\title{
FGFR inhibitor BGJ398 and HDAC inhibitor OBP-801 synergistically inhibit cell growth and induce apoptosis in bladder cancer cells
}

\author{
TOSHIYA TAKAMURA ${ }^{1,2}$, MANO HORINAKA $^{1}$, SHUSUKE YASUDA $^{1}$, SEIJIRO TORIYAMA $^{1,2}$, \\ YUICHI AONO ${ }^{1}$, YOSHIHIRO SOWA ${ }^{1}$, TSUNEHARU MIKI ${ }^{2,3}$, OSAMU UKIMURA ${ }^{2}$ and TOSHIYUKI SAKAI ${ }^{1}$ \\ Departments of ${ }^{1}$ Molecular-Targeting Cancer Prevention and ${ }^{2}$ Urology, Kyoto Prefectural University \\ of Medicine, Kyoto; ${ }^{3}$ Department of Urology, Saiseikai Shiga Hospital, Shiga, Japan
}

Received June 7, 2017; Accepted November 6, 2017

DOI: 10.3892/or.2017.6127

\begin{abstract}
In advanced bladder cancer, cisplatin-based chemotherapy has been the standard treatment for many years, but there are many problems in terms of side-effects. Recently, a number of clinical trials using molecular-targeted agents have been conducted, and new therapies are expected that could replace conventional cytotoxic chemotherapy. We herein report that concurrent treatment with fibroblast growth factor receptor (FGFR) inhibitor BGJ398 and the novel histone deacetylase (HDAC) inhibitor OBP-801/YM753/spiruchostatin A synergistically inhibited cell growth and markedly induced apoptosis in high-grade bladder cancer cells. This combination activated caspase-3, -8 and -9 , and the pan-caspase inhibitor zVAD-fmk significantly reduced the apoptotic response to the combined treatment. The combination upregulated the expression of Bim, one of the pro-apoptotic molecules. In the present study, Bim siRNA efficiently reduced apoptosis induced by the co-treatment of BGJ398 and OBP-801. Therefore, the apoptosis induced by the combination was shown to be at least partially dependent on Bim. Taken together, these results suggest that the combination of BGJ398 and OBP-801 is a novel high potential therapeutic strategy for muscle-invasive bladder cancer.
\end{abstract}

Correspondence to: Dr Mano Horinaka, Department of MolecularTargeting Cancer Prevention, Kyoto Prefectural University of Medicine, Kawaramachi-Hirokoji, Kamigyo-ku, Kyoto 602-8566, Japan

E-mail: m-hori@koto.kpu-m.ac.jp

Abbreviations: FGFR, fibroblast growth factor receptor; HDAC, histone deacetylase; MAPK, mitogen-activated protein kinase; PI3K, phosphoinositide 3-kinase; ERK, extracellular signal-related kinase; CI, combination index; FRS2, fibroblast growth factor receptor substrate 2

Key words: FGFR inhibitor, HDAC inhibitor, apoptosis, bladder cancer, Bim

\section{Introduction}

Bladder cancer is one of the most common cancers in the world, with an estimated 174,000 deaths in 2013 (1). For patients with locally advanced or metastatic bladder cancer, the standard chemotherapy is cisplatin-based combination chemotherapy. However, the 5-year survival rate in patients with stage IV bladder cancer after treatment is only around $15 \%$ (2). Considering the limited response rate of the regimens and the toxicity (3), novel therapeutic agents to replace conventional cytotoxic chemotherapy are needed.

It has been reported that genetic abnormality of various genes is observed in patients with bladder cancer. Recently, it has become clear that the signaling pathways of fibroblast growth factor receptors (FGFRs), particularly the FGFR3 (4) and FGFR1 (5) pathways, are altered in bladder cancers. FGFRs (FGFR1-FGFR4) play a key role in the regulation of proliferation, angiogenesis and apoptosis (6). The binding of FGFs to FGFRs initiates a phosphorylation cascade, resulting in signaling through various downstream pathways, including RAS-MAPK, PI3K and STATs (7). Active mutations and/or overexpression of FGFR3 are common genetic alterations in non-invasive bladder cancer, but occur at a low frequency in high-grade invasive bladder cancer (8). FGFR1 is not mutated in bladder cancer, but overexpression is frequent in bladder cancer cell lines regardless of tumor grade and stage (5). In addition, it has been reported that FGFR1 expression is an adverse prognostic risk factor in muscle invasive bladder cancer after radical cystectomy (9). Recently, a number of clinical trials of BGJ398, a selective pan FGFR inhibitor, have progressed in patients with various types of cancers. In a phase 1 clinical trial using BGJ398 in patients with solid tumors carrying FGFR genetic alternations, antitumor activity was demonstrated in patients with FGFR3-mutant bladder cancer (10). Furthermore, a study of BGJ398 in non-muscleinvasive urothelial carcinoma of the bladder is currently recruiting participants. FGFR inhibitor BGJ398 is expected to be a more effective therapeutic agent for patients with bladder cancer. However, it has been reported that BGJ398 did not suppress primary tumors, but did inhibit the development of circulating tumor cells and metastasis in vivo (11). 
Histone deacetylase (HDAC) inhibitors are also predicted for the treatment of various cancers including bladder cancer. It has been reported that HDAC inhibitors inhibit cell growth and induce cell death in bladder cancer cells (12). Furthermore, several studies have reported that HDAC inhibitors synergize with chemotherapeutic agents (13-15). Currently, a phase 2 clinical trial of an HDAC inhibitor for patients with bladder cancer is ongoing. OBP-801/YM753/spiruchostatin A was identified as a novel HDAC inhibitor by using a p21 promoterreporter assay, which showed the most potent HDAC-inhibitory activity as compared to other HDAC inhibitors (16).

We showed for the first time that a combined treatment of FGFR inhibitor BGJ398 and HDAC inhibitor OBP-801 synergistically inhibited cell growth, and induced apoptosis in human bladder cancer cells. Taken together, we suggest that the combination is a promising novel therapeutic approach to treat muscle-invasive bladder cancer.

\section{Materials and methods}

Cell culture. Human bladder cancer UM-UC-3 and T24 cells were purchased from the American Type Culture Collection (ATCC; Manassas, VA, USA) and cultured in RPMI-1640 medium supplemented with $10 \%$ fetal bovine serum (FBS), $2 \mathrm{mM}$ L-glutamine, $100 \mathrm{U} / \mathrm{ml}$ penicillin and $100 \mu \mathrm{g} / \mathrm{ml} \mathrm{strep-}$ tomycin. Cell cultures were incubated at $37^{\circ} \mathrm{C}$ in a humidified atmosphere of $5 \% \mathrm{CO}_{2}$.

Reagents. BGJ398 was purchased from Selleck Chemicals (Houston, TX, USA). OBP-801 was supplied by Oncolys BioPharma (Tokyo, Japan). zVAD-fmk was purchased from R\&D Systems (Minneapolis, MN, USA). BGJ398, OBP-801 and zVAD-fmk were dissolved in dimethyl sulfoxide (DMSO).

Cell viability assay. The number of viable cells was determined using the Cell Counting Kit-8 (CCK-8) assay according to the manufacturer's instructions (Dojindo Laboratories, Kumamoto, Japan). After the incubation of cells $\left(3 \times 10^{3} /\right.$ well) in 96-well plates for $72 \mathrm{~h}$ with the indicated concentrations of BGJ398 or OBP-801, the kit reagent was added to the medium, and the plates were incubated for a further $4 \mathrm{~h}$. The absorbance of samples $(450 \mathrm{~nm})$ was determined using a scanning multiwell spectrophotometer (DS Pharma Biomedical, Co., Ltd., Osaka, Japan).

Combination index. Combination index (CI) values were analyzed using CalcuSyn software (Biosoft, Great Shelford, UK). CI $<1$ indicates synergism greater than the expected additive effect.

Detection of apoptosis. After the incubation of cells $\left(2 \times 10^{4} /\right.$ well) in 6-well plates for $72 \mathrm{~h}$ with the agents, the cells were harvested.After washing with phosphate-buffered saline (PBS), the cells were treated with PBS containing $0.1 \%$ Triton X-100 and the nuclei were stained with propidium iodide (PI; Sigma-Aldrich, St. Louis, MO, USA). The DNA content was measured using a FACSCalibur (Becton-Dickinson, Franklin Lakes, NJ, USA). CellQuest software (Becton-Dickinson) was used to analyze the data. DNA fragmentation was quantified by the percentage of hypodiploid DNA (sub-G1).
Western blotting. Western blot analysis was carried out as previously described (17). The following antibodies were purchased from the indicated sources: rabbit monoclonal antibodies for anti-Bim (ab32158) (Abcam, Cambridge, UK), anti-Bcl-xL (\#2762), and anti-PARP (\#9542) (Cell Signaling Technology, Beverly, MA, USA); rabbit polyclonal antibodies for antisurvivin (AF886) (R\&D Systems), anti-DR5 (\#8074) (Cell Signaling Technology), anti-DR4 (1139) (ProSci, Inc., Poway, CA, USA), anti-caspase-3 (\#9665), anti-histone H4 (\#2935), anti-acetyl-histone H4 (\#9672), anti-Bid (\#2002), anti-ERK1/2 (\#9102), anti-Shp2 (\#3752), anti-phospho ERK1/2 (\#9101), anti-phospho FRS2- $\alpha$ (\#3861), anti-phospho Shp2 (\#3751) (Cell Signaling Technology), anti-FRS2- $\alpha$ (SC17841) (Santa Cruz Biotechnology, Dallas, TX, USA); mouse monoclonal antibodies for anti-caspase-8 (M032-3) and anti-caspase-9 (M054-3) (MBL, Nagoya, Japan), anti-FLIP (ACX-804-961-0100) (Enzo Life Sciences, Farmingdale, NY, USA), and anti-GAPDH (5G4) (HyTest, Ltd., Turku, Finland) were used as primary antibodies. An anti-rabbit IgG-HRP-conjugated antibody (NA934) (GE Healthcare Life Sciences, Little Chalfont, UK) and an antimouse IgG-HRP-conjugated antibody (NA931) (GE Healthcare Life Sciences) were used as the secondary antibodies. The signal was detected using the Chemilumi-One chemiluminescent kit (Nacalai Tesque, Inc., Kyoto, Japan) or chemiluminescent HRP substrate (Millipore, Billerica, MA, USA).

Small interfering RNA transfection. The Bim siRNA and the negative control siRNA were purchased from Sigma-Aldrich. The Bim siRNA (ACUUACAUCAGAAGGUUGC) was used for the transfection. At the same time as the transfection, UM-UC-3 and T24 cells ( $2 \times 10^{4} /$ well) were seeded in 6-well plates without antibiotics. The Bim siRNA ( $5 \mathrm{nM}$ for UM-UC-3 cells or $10 \mathrm{nM}$ for T24 cells) was transfected into cells using Lipofectamine RNAiMAX (Invitrogen, Carlsbad, CA, USA) according to the manufacturer's instructions. Twenty-four hours after the transfection, the cells were treated with agents for $48 \mathrm{~h}$ and then harvested.

Statistical analysis. Data are expressed as the means \pm SD of three determinations. Statistical analysis was performed using a Student's t-test. Samples were considered significantly different at $\mathrm{P}<0.05$.

\section{Results}

Combined treatment with BGJ398 and OBP-801 synergistically inhibits cell growth in human bladder cancer cells. To examine the anti-growth effects of BGJ398 or OBP-801 alone against human bladder cancer cells, we first assessed the viable cell number at $72 \mathrm{~h}$ after treatment with the indicated concentrations of the agents. Each agent suppressed the cell growth of UM-UC-3 and T24 cells in a dose-dependent manner (Fig. 1A). Notably, co-treatment with BGJ398 and OBP-801 markedly inhibited cell growth, compared with the single treatment of each agent in both cell lines (Fig. 1B). Moreover, the combination index (CI) values for BGJ398 and OBP-801 were $<1.0$, indicating a synergistic effect on the inhibition of cell growth (Table I).

We next examined the effects of the combined treatment of FGFR inhibitor BGJ398 and HDAC inhibitor OBP-801 
A

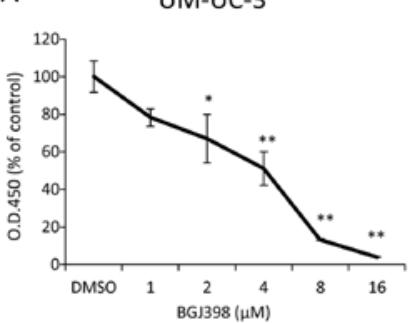

UM-UC-3
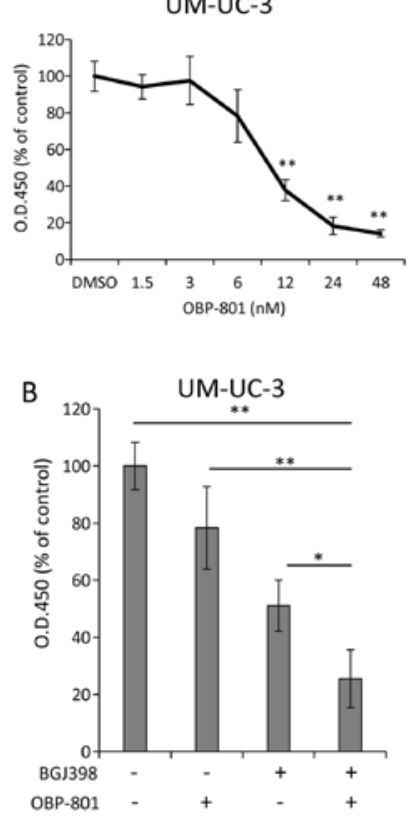

T24

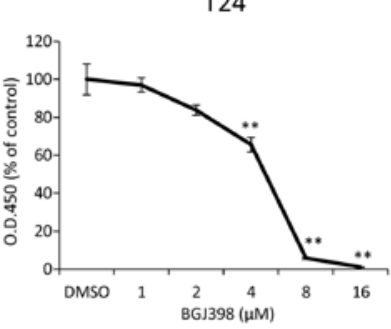

T24
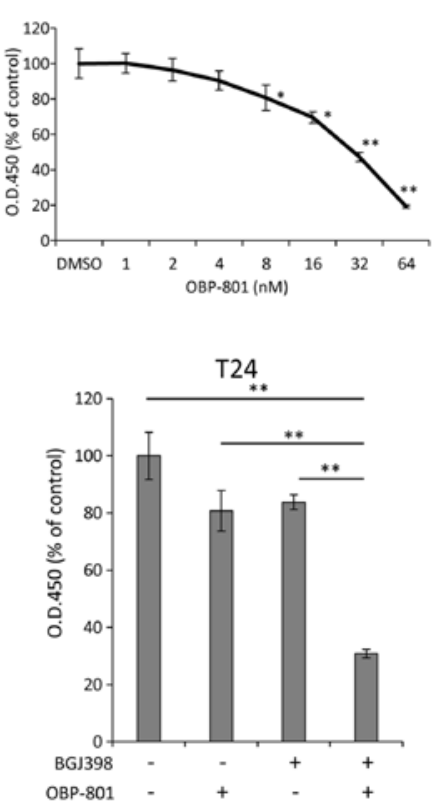

Figure 1. BGJ398 and OBP-801 synergistically inhibit the cell growth of human bladder cancer cells. (A) Human bladder cancer UM-UC-3 and T24 cells were treated with BGJ398 and OBP-801 at the indicated concentrations. After incubation for $72 \mathrm{~h}$, cell viability was assessed by CCK-8 assay. Columns, means of triplicate data; bars, SD; ${ }^{*} \mathrm{P}<0.05,{ }^{* *} \mathrm{P}<0.01$. (B) UM-UC- 3 cells were treated with $4 \mu \mathrm{M}$ BGJ398 and/or $6 \mathrm{nM}$ OBP-801. T24 cells were treated with $2 \mu \mathrm{M}$ BGJ398 and/or $8 \mathrm{nM}$ OBP-801. After incubation for $72 \mathrm{~h}$, cell viability was examined by CCK- 8 assay. Columns, means of triplicate data; bars, $\mathrm{SD} ;{ }^{*} \mathrm{P}<0.05,{ }^{* *} \mathrm{P}<0.01$.

Table I. The combination index of BGJ398 and OBP-801 was calculated in the UM-UC-3 and T24 cells.

\begin{tabular}{lcc}
\hline BGJ398 $(\mu \mathrm{M})$ & OBP-801 $(\mathrm{nM})$ & Combination index \\
\hline UM-UC-3 & UM-UC-3 & \\
2 & 3 & 2.098 \\
4 & 6 & 0.669 \\
8 & 12 & 0.216 \\
T24 & T24 & \\
1 & 4 & 1.030 \\
2 & 8 & 0.591 \\
4 & 16 & 0.259 \\
\hline
\end{tabular}

on the FGFR signaling pathways and HDAC-inhibitory activity. OBP-801 alone and the combination increased the acetylation of histone $\mathrm{H} 4$ in both UM-UC-3 and T24 cells (Fig. 2A). BGJ398 alone and the combination inhibited

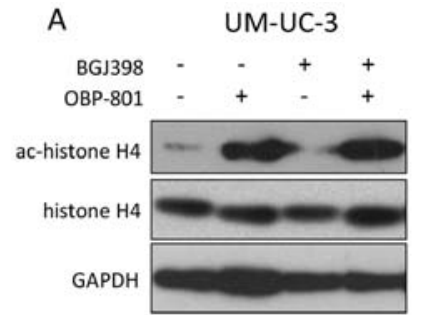

B
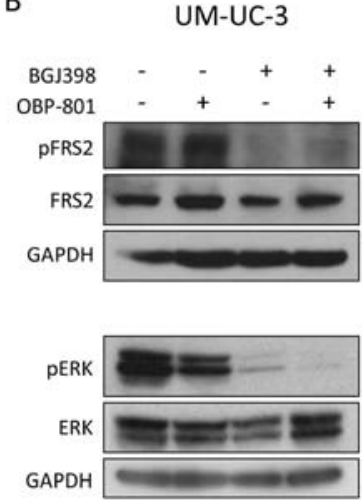

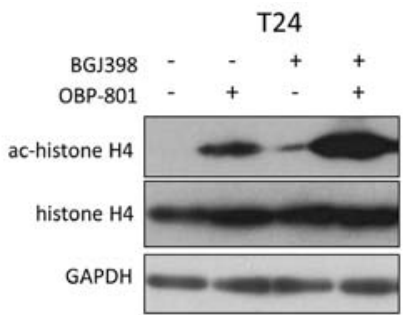

T24
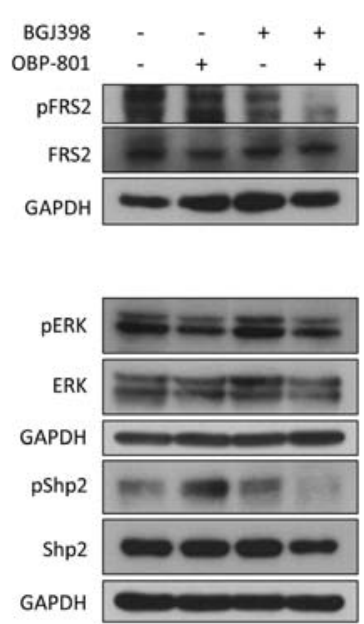

Figure 2. Effects of the combined treatment of BGJ398 and OBP-801 on the acetylation of histone $\mathrm{H} 4$ and FGFR signaling pathways. (A) Western blotting of anti-histone $\mathrm{H} 4$, anti-acetyl-histone $\mathrm{H} 4$. GAPDH served as a loading control in all blots. UM-UC-3 cells were treated with $4 \mu \mathrm{M}$ BGJ398 and/or $6 \mathrm{nM}$ OBP- 801 for $48 \mathrm{~h}$. T2 24 cells were treated with $2 \mu \mathrm{M}$ BGJ398 and/or $8 \mathrm{nM}$ OBP-801 for $48 \mathrm{~h}$. (B) Western blotting of anti-phospho FRS2- $\alpha$, antiFRS2- $\alpha$, anti-phospho ERK1/2, anti-ERK1/2 in UM-UC-3 cells. Western blotting of anti-phospho FRS2- $\alpha$, anti-FRS2- $\alpha$, anti-phospho ERK1/2, antiERK1/2, anti-phospho Shp2 and anti-Shp2 in T24 cells. GAPDH served as a loading control in all blots.

the phosphorylation of FGFR substrate 2 (FRS2), which is a docking protein linking FGFRs, in UM-UC-3 cells. Furthermore, BGJ398 also inhibited the phosphorylation of ERK in UM-UC-3 cells (Fig. 2B). On the other hand, BGJ398 slightly inhibited phosphorylation of FRS2, and the co-treatment of OBP-801 and BGJ398 more clearly inhibited it in T24 cells. However, the treatment of BGJ398 alone and the combination did not inhibit the phosphorylation of ERK in T24 cells (Fig. 2B). Therefore, we next examined the effect of the combination on the phosphorylation of protein tyrosine phosphatase Shp2. Shp2 is a critical downstream mediator of FGFR-FRS2 signaling. As shown in Fig. 2B, the combined treatment inhibited the phosphorylation of Shp2 in T24 cells.

Combined treatment with BGJ398 and OBP-801 induces caspase-dependent apoptosis in human bladder cancer cells. To clarify the mechanisms of the synergistic inhibitory effects on cell growth of bladder cancer cells by the combined treatment with BGJ398 and OBP-801, we next investigated the effect of the combination on apoptosis by measuring the sub-G1 populations, using flow cytometry after treatment for $72 \mathrm{~h}$. The co-treatment with BGJ398 and OBP-801 more markedly induced apoptosis than that of each agent alone in the UM-UC-3 and T24 cells (Fig. 3). To examine whether the apoptosis induced by the combination of the agents is caspase-dependent, 
A

UM-UC-3

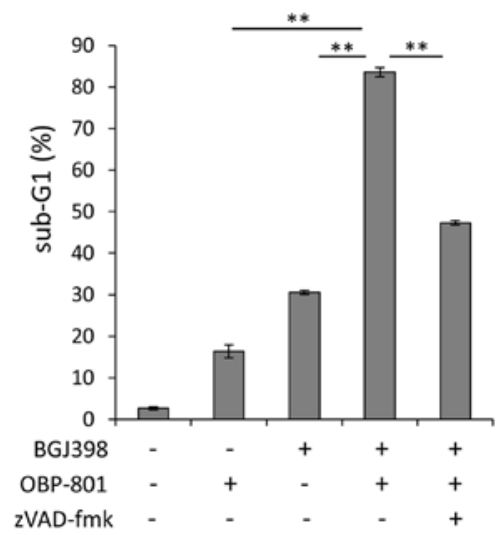

B $\mathrm{T} 24$

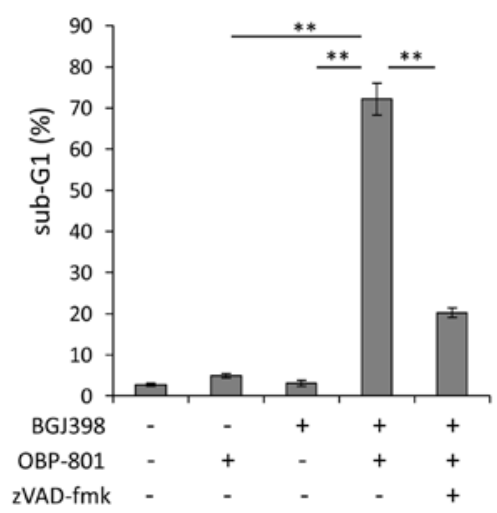

Figure 3. Combination treatment with BGJ398 and OBP-801 induces caspase-dependent apoptosis in human bladder cancer cells. (A) UM-UC-3 cells were treated with $4 \mu \mathrm{M}$ BGJ398 and/or $6 \mathrm{nM}$ OBP-801 with or without the pan-caspase inhibitor zVAD-fmk $(20 \mu \mathrm{M})$ for $72 \mathrm{~h}$. The apoptotic cell population was analyzed by flow cytometry. Columns, means of triplicate data; bars, SD; ${ }^{* *} \mathrm{P}<0.01$. (B) T24 cells were treated with $2 \mu \mathrm{M}$ BGJ398 and/or $8 \mathrm{nM}$ OBP-801 with or without the pancaspase inhibitor zVAD-fmk $(20 \mu \mathrm{M})$ for $72 \mathrm{~h}$. The apoptotic cell population was analyzed by flow cytometry. Columns, means of triplicate data; bars, SD; ${ }^{* *} \mathrm{P}<0.01$.

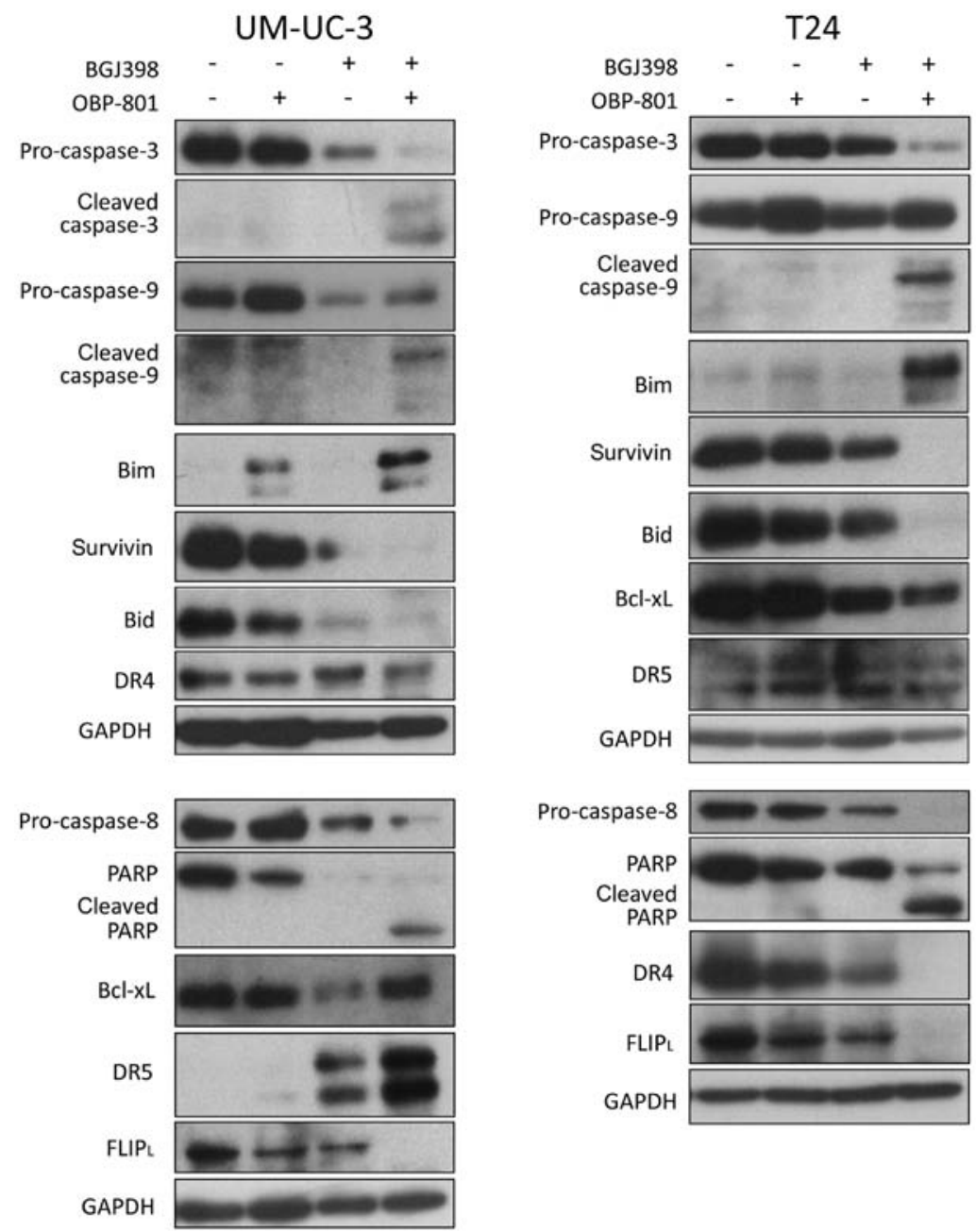

Figure 4. Combined treatment with BGJ398 and OBP-801 induces caspase-dependent apoptosis in human bladder cancer cells through both intrinsic and extrinsic pathways. Western blotting of caspase-3, -8, -9, PARP, Bim, survivin, Bid, Bcl-xL, DR5, DR4 and FLIP L $_{\mathrm{L}}$ in UM-UC-3 and T24 cells. UM-UC-3 cells were treated with $4 \mu \mathrm{M}$ BGJ398 and/or $6 \mathrm{nM}$ OBP-801 for $48 \mathrm{~h}$. T2 4 cells were treated with $2 \mu \mathrm{M}$ BGJ398 and/or $8 \mathrm{nM}$ OBP-801 for $48 \mathrm{~h}$. GAPDH served as a loading control in all blots.

we analyzed the effect of a caspase inhibitor. The pan-caspase inhibitor zVAD-fmk effectively blocked apoptosis induced by the co-treatment with BGJ398 and OBP-801 (Fig. 3). These results suggest that the combination of BGJ398 and OBP-801 synergistically induces caspase-dependent apoptosis in human bladder cancer cells. 
A
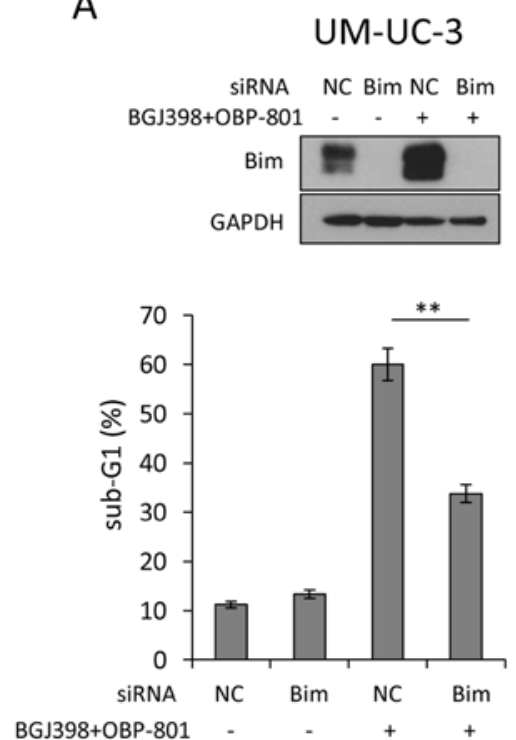

B

\section{T24}
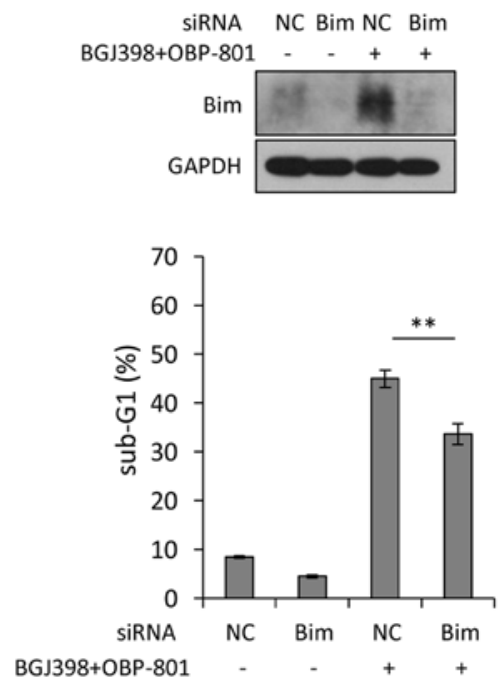

Figure 5. Bim contributes to apoptosis by combined treatment with BGJ398 and OBP-801 in human bladder cancer cells. (A) UM-UC-3 cells were transfected with $5 \mathrm{nM}$ Bim siRNA or NC (negative control) siRNA. After $24 \mathrm{~h}$, the cells were incubated with $2 \mu \mathrm{M} \mathrm{BGJ} 398$ and/or $8 \mathrm{nM}$ OBP-801 for $48 \mathrm{~h}$. Western blotting of Bim was performed. GAPDH served as a loading control. The apoptotic cell population was analyzed by flow cytometry. Columns, means of triplicate data; bars, SD; ${ }^{* *} \mathrm{P}<0.01$. (B) T24 cells were transfected with $10 \mathrm{nM}$ Bim siRNA or NC siRNA. After $24 \mathrm{~h}$, the cells were incubated with $2 \mu \mathrm{M}$ BGJ398 and/or $8 \mathrm{nM}$ OBP-801 for $48 \mathrm{~h}$. Western blotting of Bim was performed. GAPDH was a loading control. The apoptosis population was analyzed by flow cytometry. Columns, means of triplicate data; bars, $\mathrm{SD} ;{ }^{* *} \mathrm{P}<0.01$.

Combined treatment with BGJ398 and OBP-801 causes apoptosis via intrinsic and extrinsic pathways in human bladder cancer cells. We examined the expression of apoptosis-related proteins in the combined treatment. As shown in Fig. 4, the concurrent treatment induced the cleavage of caspase- 8 , caspase-9, caspase-3 and PARP. The co-treatment increased the expression of pro-apoptotic protein Bim, and reduced the expression of anti-apoptotic proteins, survivin and FLIP $_{\mathrm{L}}$, in UM-UC-3 and T24 cells (Fig. 4). The expression of DR5, one of the pro-apoptotic proteins, was upregulated by co-treatment in UM-UC-3 cells, but not in T24 cells. These results suggest that the combined treatment with BGJ398 and OBP-801 induced apoptosis through the activation of both intrinsic and extrinsic pathways in human bladder cancer cells.

Bim contributes to apoptosis by the combined treatment in bladder cancer cells. The combined treatment upregulated the expression of Bim in the UM-UC-3 and T24 cells (Fig. 4). We examined whether Bim contributed to the induction of apoptosis by co-treatment with BGJ398 and OBP-801. The effects of the Bim knockdown were confirmed by western blotting (Fig. 5). Bim siRNA significantly suppressed apoptosis induced by the combination compared with the control (Fig. 5). These results suggest that the combined treatment with BGJ398 and OBP-801 caused apoptosis at least partially through the upregulation of Bim in bladder cancer cells.

\section{Discussion}

In the present study, we showed for the first time the synergistic effect of the combined treatment with FGFR inhibitor BGJ398 and the novel HDAC inhibitor OBP-801 on cell growth arrest and apoptosis against high grade bladder cancer cells. Furthermore, the apoptosis was associated with the upregulation of Bim and DR5, and downregulation of survivin and FLIP by the combined treatment as the molecular mechanisms. In these apoptosis-related proteins, we showed that Bim knockdown significantly inhibited apoptosis induced by the combination (Fig. 5). The results suggest that the combined treatment causes apoptosis at least partially through the upregulation of Bim in bladder cancer cells.

$\mathrm{Bim}$ is a BH3-only pro-apoptotic member of the $\mathrm{Bcl}-2$ family (18), and its expression is regulated by ERK1/2 and PI3K/ AKT pathways (19). The activation of the ERK1/2 pathway induces the phosphorylation of Bim and promotes proteasomedependent degradation of phosphorylated Bim (20). In the present study, OBP-801 slightly inhibited the phosphorylation of ERK1/2 in UM-UC-3 cells (Fig. 2B). A previous report showed that HDAC inhibitors decreased phosphorylation of ERK by upregulating dual-specificity phosphatase (DUSP) (21). DUSP may be involved in the downregulation of ERK1/2 by OBP-801 in UM-UC-3 cells. On the other hand, the effect of BGJ398 was different in the UM-UC-3 and in T24 cells. BGJ398 clearly inhibited the phosphorylation of ERK1/2 in UM-UC-3 cells (Fig. 2B). The phosphorylation of ERK1/2 was more clearly inhibited by the co-treatment of BGJ398 and OBP-801 in UM-UC-3 cells (Fig. 2B). These results suggest that the expression of Bim was upregulated by the combination in ERK1/2-dependent pathways in UM-UC-3 cells.

In the present study, BGJ398 alone and co-treatment with OBP-801 inhibited the phosphorylation of FRS2 in T24 cells (Fig. 2B). On the other hand, the phosphorylation of ERK was not suppressed by these treatments in T24 cells (Fig. 2B). Therefore, we next examined whether the combination regulated Shp2 downstream of FGFR-FRS2 signaling independent of ERK. Shp2, a non-receptor phosphotyrosine phosphatase, promotes tumor progression in various cancer types (22). Furthermore, previous studies reported that Shp2 is a key 
downregulator of Bim through ERK1/2 activation (23,24). Notably, in this study, the combination inhibited phosphorylation of Shp2 but not the phosphorylation of ERK in T24 cells. These data showed that Bim was upregulated by Shp2 independent of ERK in T24 cells treated by the combination, suggesting the existence of an unknown pathway between Shp2 and Bim independent of ERK.

Moreover, the expression of DR5 was upregulated by the combination in UM-UC-3 cells (Fig. 4). We previously reported that OBP-801 and celecoxib synergistically induced apoptosis via the DR5-dependent pathway, and that Bim partially acts as one of the key molecules downstream of DR5 in bladder cancer cells (17). Therefore, the DR5-dependent pathway may be partially involved in the caspase-dependent apoptosis by the combination of BGJ398 and OBP-801 in UM-UC-3 cells.

In conclusion, this is the first report showing the synergistic effect of the combination of an FGFR inhibitor and an HDAC inhibitor against human bladder cancer cells. We suggest that the combined treatment is a promising novel therapeutic approach to treat muscle-invasive bladder cancer.

\section{Acknowledgements}

The present study was supported by the Research Funding of Kyoto Prefectural University of Medicine.

\section{References}

1. Fitzmaurice C, Dicker D, Pain A, Hamavid H, Moradi-Lakeh M, MacIntyre MF, Allen C, Hansen G, Woodbrook R, Wolfe C, et al; Global Burden of Disease Cancer Collaboration: The Global Burden of Cancer 2013. JAMA Oncol 1: 505-527, 2015.

2. von der Maase H, Sengelov L, Roberts JT, Ricci S, Dogliotti L, Oliver T, Moore MJ, Zimmermann A and Arning M: Long-term survival results of a randomized trial comparing gemcitabine plus cisplatin, with methotrexate, vinblastine, doxorubicin, plus cisplatin in patients with bladder cancer. J Clin Oncol 23: 4602-4608, 2005.

3. Drayton RM and Catto JW: Molecular mechanisms of cisplatin resistance in bladder cancer. Expert Rev Anticancer Ther 12 : 271-281, 2012.

4. Tomlinson DC, Baldo O, Harnden P and Knowles MA: FGFR3 protein expression and its relationship to mutation status and prognostic variables in bladder cancer. J Pathol 213: 91-98, 2007.

5. Tomlinson DC, Lamont FR, Shnyder SD and Knowles MA: Fibroblast growth factor receptor 1 promotes proliferation and survival via activation of the mitogen-activated protein kinase pathway in bladder cancer. Cancer Res 69: 4613-4620, 2009.

6 . Turner $\mathrm{N}$ and Grose R: Fibroblast growth factor signalling: From development to cancer. Nat Rev Cancer 10: 116-129, 2010.

7. Klint $\mathrm{P}$ and Claesson-Welsh L: Signal transduction by fibroblast growth factor receptors. Front Biosci 4: D165-D177, 1999.

8. Cancer Genome Atlas Research Network: Comprehensive molecular characterization of urothelial bladder carcinoma. Nature 507: 315-322, 2014.

9. Lim S, Koh MJ, Jeong HJ, Cho NH, Choi YD, Cho Y, Lee HY and Rha SY: Fibroblast growth factor receptor 1 overexpression is associated with poor survival in patients with resected muscle invasive urothelial carcinoma. Yonsei Med J 57: 831-839, 2016.
10. Nogova L, Sequist LV, Perez Garcia JM, Andre F, Delord JP, Hidalgo M, Schellens JH, Cassier PA, Camidge DR, Schuler M, et al: Evaluation of BGJ398, a fibroblast growth factor receptor 1-3 kinase inhibitor, in patients with advanced solid tumors harboring genetic alterations in fibroblast growth factor receptors: Results of a global phase I, dose-escalation and dose-expansion study. J Clin Oncol 35: 157-165, 2017.

11. Cheng T, Roth B, Choi W, Black PC, Dinney C and McConkey DJ: Fibroblast growth factor receptors-1 and -3 play distinct roles in the regulation of bladder cancer growth and metastasis: Implications for therapeutic targeting. PLoS One 8: e57284, 2013.

12. Li QQ, Hao JJ, Zhang Z, Hsu I, Liu Y, Tao Z, Lewi K, Metwalli AR and Agarwal PK: Histone deacetylase inhibitor-induced cell death in bladder cancer is associated with chromatin modification and modifying protein expression: A proteomic approach. Int J Oncol 48: 2591-2607, 2016.

13. Jeon HG, Yoon CY, Yu JH, Park MJ, Lee JE, Jeong SJ, Hong SK, Byun SS and Lee SE: Induction of caspase mediated apoptosis and down-regulation of nuclear factor- $\mathrm{kB}$ and Akt signaling are involved in the synergistic antitumor effect of gemcitabine and the histone deacetylase inhibitor trichostatin $\mathrm{A}$ in human bladder cancer cells. J Urol 186: 2084-2093, 2011.

14. Wang D, Jing Y, Ouyang S, Liu B, Zhu T, Niu H and Tian Y: Inhibitory effect of valproic acid on bladder cancer in combination with chemotherapeutic agents in vitro and in vivo. Oncol Lett 6: 1492-1498, 2013.

15. Li DR, Zhang H, Peek E, Wang S, Du L, Li G and Chin AI: Synergy of histone-deacetylase inhibitor AR-42 with cisplatin in bladder cancer. J Urol 194: 547-555, 2015.

16. Shindoh N, Mori M, Terada Y, Oda K, Amino N, Kita A, Taniguchi M, Sohda KY, Nagai K, Sowa Y, et al: YM753, a novel histone deacetylase inhibitor, exhibits antitumor activity with selective, sustained accumulation of acetylated histones in tumors in the WiDr xenograft model. Int J Oncol 32: 545-555, 2008.

17. Toriyama S, Horinaka M, Yasuda S, Taniguchi T, Aono Y, Takamura T, Morioka Y, Miki T, Ukimura O and Sakai T: A histone deacetylase inhibitor, OBP-801, and celecoxib synergistically inhibit the cell growth with apoptosis via a DR5-dependent pathway in bladder cancer cells. Mol Cancer Ther 15: 2066-2075, 2016.

18. Youle RJ and Strasser A: The BCL-2 protein family: Opposing activities that mediate cell death. Nat Rev Mol Cell Biol 9: 47-59, 2008.

19. Balmanno $\mathrm{K}$ and Cook SJ: Tumour cell survival signalling by the ERK1/2 pathway. Cell Death Differ 16: 368-377, 2009.

20. Ley R, Balmanno K, Hadfield K, Weston C and Cook SJ: Activation of the ERK1/2 signaling pathway promotes phosphorylation and proteasome-dependent degradation of the $\mathrm{BH} 3$-only protein, Bim. J Biol Chem 278: 18811-18816, 2003.

21. Ferguson BS, Harrison BC, Jeong MY, Reid BG, Wempe MF, Wagner FF, Holson EB and McKinsey TA: Signal-dependent repression of DUSP5 by class I HDACs controls nuclear ERK activity and cardiomyocyte hypertrophy. Proc Natl Acad Sci USA 110: 9806-9811, 2013.

22. Zhang J, Zhang F and Niu R: Functions of Shp2 in cancer. J Cell Mol Med 19: 2075-2083, 2015.

23. Yang W, Klaman LD, Chen B, Araki T, Harada H, Thomas SM, George EL and Neel BG: An Shp2/SFK/Ras/Erk signaling pathway controls trophoblast stem cell survival. Dev Cell 10: 317-327, 2006.

24. Selimoglu-Buet D, Gallais I, Denis N, Guillouf C and MoreauGachelin F: Oncogenic kit triggers Shp2/Erk1/2 pathway to down-regulate the pro-apoptotic protein Bim and to promote apoptosis resistance in leukemic cells. PLoS One 7: e49052, 2012. 